\title{
LA UNIVERSIDAD COMO AGENTE DINAMIZADOR DEL DESARROLLO TERRITORIAL: EL CASO DE LA UNIVERSIDAD DE CÁDIZ Y EL SECTOR AERONÁUTICO EN ESPAÑA
}

Susana Reyes-Benítez

Universidad de Cádiz

España 
Panorama Económico, Vol. 25 - No. 3 (Julio - Septiembre de 2017), pp. 399-422

\title{
Susana Reyes-Benítez
}

\section{La Universidad como agente dinamizador del desarrollo territorial: el caso de la Universidad de Cádiz y el sector aeronáutico en España}

\begin{abstract}
Resumen como el actual.

Palabras clave: Universidad, Desarrollo territorial, Aeronaútica, Cádiz.

Clasificación JEL: I20, I21, I25, H75
\end{abstract}

Tradicionalmente los modelos de crecimiento económico no incluían el capital humano en su análisis, centrándose en la evolución del ahorro, la inversión, la demografía, entre otros. Sin embargo, a finales del siglo XX, se determinó que era necesario incluir el capital humano en los modelos de crecimiento económico, como uno de los factores más valiosos que contribuyen a dinamizar el desarrollo territorial. En este artículo, se pretende evidenciar el papel de la Universidad de Cádiz como agente dinamizador del sistema productivo local del sector aeronáutico en la provincia de Cádiz. Los resultados buscan facilitar el establecimiento de mecanismos y orientar la implementación de nuevos proyectos de colaboración entre la universidad y el sector de forma que se pueda minimizar el impacto sobre el mismo durante periodos de crisis económica

\section{The University as a driving force for territorial development: the case of the Universidad de Cadiz and the aeronautical sector in Spain}

\begin{abstract}
Traditionally, economic growth models did not include human capital among its variables, focusing on other factors such as changes in savings, investment, demographics, and so on. However, at the end of the twentieth century, it was determined that it was necessary to include human capital in the models of economic growth, because it is one of the most valuable factors contributing to the dynamisation of development. In this article, we intend to demonstrate the role of the University of Cádiz as a dynamic agent of the local production system of the aeronautical sector in the province of Cádiz. The results can facilitate the setting up of mechanisms and guide the implementation of new collaborative projects between the university and the sector, so as to minimize their impact in times of economic crisis such as the current one. Keywords: University, Territorial development, Aeronautics, Cádiz.
\end{abstract}

JEL Classification: I20, I21, I25, H75

\section{L'université en tant que moteur du développement territorial: le cas de l'Université de Cadix et du secteur aéronautique en Espagne}

\begin{abstract}
Résumé
Traditionnellement, les modèles de croissance économique n'incluaient pas le capital humain parmi ses variables, se concentrant sur d'autres facteurs tels que l'évolution de l'épargne, l'investissement, la démographie, etc. Cependant, à la fin du XXe siècle, il a été déterminé qu'il était nécessaire d'inclure le capital humain dans les modèles de croissance économique, car c'est l'un des facteurs les plus précieux qui contribuent à la dynamisation du développement territorial. Dans cet article, nous avons l'intention de démontrer le rôle de l'Université de Cádiz en tant qu'agent dynamique du système de production local du secteur aéronautique dans la province de Cádiz. Les informations obtenues peuvent faciliter la mise en place de mécanismes et orienter la mise en œuvre de nouveaux projets collaboratifs entre l'université et le secteur, de manière à en minimiser l'impact en période de crise économique telle que celle en cours.

Mots-clés: Université, Développement territorial, Aéronautique, Cadix.
\end{abstract}

Nomenclature JEL: I20, I21, I25, H75 


\section{La Universidad como agente dinamizador del desarrollo territorial: el caso de la Universidad de Cádiz y el sector aeronáutico en España}

INFORMACIÓN DEL ARTÍCULO

Recepción de artículo: 08/04/2017

Concepto de evaluación: 02/05/2017

Aceptación de artículo: 16/06/2017
Susana Reyes-Benítez*

Universidad de Cádiz

España

\section{INTRODUCCIÓN}

Las evidencias empíricas y los enfoques teóricos más recientes de la economía regional ligados a los conceptos de "medio innovador", "distrito industrial" o "sistema productivo local", ponen de manifiesto que el desarrollo regional, en un entorno de creciente competitividad en los mercados, depende de la capacidad de propiciar la innovación en áreas geográficas concretas.

Con este artículo, se pretende evidenciar ese papel de la Universidad de Cádiz como agente dinamizador del sistema productivo local del sector aeronáutico en la provincia de Cádiz, mediante los acuerdos y colaboraciones con las empresas para que se facilite la trasferencia de conocimientos desde la universidad hacia el sector productivo. En definitiva, el objetivo general de este artículo es caracterizar del papel de la Universidad de Cádiz como agente de desarrollo territorial. Adicionalmente como objetivos específicos se establecen los siguientes: por un lado caracterizar la importancia sector aeronáutico en la provincia de Cádiz. Con este artículo se pretende ofrecer una visión concreta de la influencia de la Universidad de Cádiz en el sector aeronáutico en los primeros años del siglo XXI.

El período seleccionado para realizar el estudio es la primera década del siglo XXI. Debido a que es la década del inicio del desarrollo del sector en Andalucía.

Se ha optado por una metodología de carácter deductivo-demostrativo que, tras una breve referencia a la información disponible, comienza por revisar con cierta amplitud

\footnotetext{
* Autor para correspondencia

Correo electrónico: sreyesbenitez@hotmail.com
} 
los conceptos y el debate teórico que constituye el marco de referencia en el que se integra la investigación. Para el marco teórico se referencia todo el material consultado en la bibliografía. Adicionalmente destacar la consulta de datos que recopilan los informes anuales de la fundación Hélice, tal fundación ofrece un servicio público de recopilación de datos accesibles a través de sus informes o página web, relativos a parámetros como: facturación, número empleados, número de empresas, tipos de empleo, tipos de empleados, etc. Por último, se han utilizado datos publicados de la relación Universidad y las empresas del sector aeronáutico, las fuentes están detalladas también en la bibliografía.

\section{MARCO TEÓRICO}

Se ha considerado los siguientes 4 conceptos como claves para poder desarrollar un marco teórico adecuado para explicar el papel de la universidad de Cádiz como agente dinamizador del Desarrollo Territorial gracias a su colaboración con el sector aeronáutico. Inicialmente es necesario una pequeña introducción de los conceptos de innovación, territorio y su relación, posteriormente se continúa avanzando para definir el concepto de sistemas productivos locales, como por ejemplo tendríamos en el caso de la red empresarial del sector en la provincia. En tercer lugar, se introduce el concepto de medio innovador para introducir conceptos que veremos tiene la relación Universidad y el sector, finalmente se termina con el concepto de como esas redes de cooperación se convierten en dinamizadores de la generación de medios innovadores. Los cuatro aspectos indicados serían:

- Innovación y territorio.

- Redesempresarialesyespaciosemergentes: los sistemas productivos locales
- El territorio como protagonista de la innovación: los medios o entornos innovadores

- Las redes de cooperación como principio generador de medios innovadores

\subsubsection{Innovación y territorio.}

El análisis de las teorías que intentan explicar la localización industrial en el espacio comienza con Weber (1929); según este autor, la ubicación de una planta industrial está relacionada con cuatro factores fundamentales: la distancia a los recursos naturales, la distancia al mercado, los costes de la mano de obra y las economías de aglomeración. Estas últimas permiten beneficiarse del acceso común a los mercados y vías de comunicación, a los servicios, a la mano de obra especializada y a los proveedores. La descentralización de actividades y la especialización flexible, confiriendo un nuevo protagonismo a las Pymes y al desarrollo regional. Así se revitalizan conceptos como los «Distritos industriales», y surgen otros nuevos como los «Medios innovadores» (Aydalot, 1986).

La innovación es un proceso social, inmerso en un contexto económico, cultural, institucional y territorial que la explica (Morgan 1997; Asheim y Dunford, 1997). Por tanto, la forma en que los territorios utilizan sus recursos patrimoniales es determinante para poder considerarlos innovadores (Caravaca et al., 2003). Los conceptos innovación y territorio, se puede relacionarlos indicando que la innovación en cierta forma busca el desarrollo territorial integrado. Se entiende por integrado aquel que es capaz de hacer compatible la competitividad económica, el bienestar social, la sostenibilidad ambiental y la moderación de los desequilibrios.

Respecto a las estrategias de desarrollo del territorio, se observa la erupción de 
estrategias locales y regionales frente a las estatales genéricas. Es decir, el concepto de desarrollo se aleja del de simple crecimiento de los indicadores macro. Dentro de esos actores, se considera vital un buen funcionamiento de las empresas de la región, capaz de asegurar la generación de ventajas competitivas en mercados cada vez más abiertos, continúa manteniendo una importancia de primer orden por diversas razones, de las que se puede destacar que son generadoras de rentas, empleos y bienes.

Una vez introducidos los conceptos de innovación-territorio y resaltando el factor geográfico como clave en el estudio de las estrategias de desarrollo territorial, es conveniente continuar en el marco teórico desarrollando los sistemas productivos locales y su papel fundamental en el desarrollo territorial.

\subsubsection{Redes empresariales y espacios emergentes: los sistemas productivos locales}

Según Méndez,R.-Caravaca,I., (1996), dentro de la diversidad de situaciones y trayectorias que muestran los estudios de casos, se ha decantado una caracterización del sistema productivo local/distrito industrial que puede sintetizarse en los siguientes componentes

- Concentración de PYMES surgidas prioritariamente por iniciativa local en un área de dimensiones reducidas.

- Especialización en una rama industrial o en torno a un tipo de producto. (Imagen de marca)

- Intensa división interempresarial del trabajo. (Redes locales, impulsoras de innovación)

- Existencia de relaciones de cooperación/solidaridad. (Asociaciones empresariales, institutos tecnológicos, centros de empresas...)

- Existencia un alto grado de flexibilidad, (mercado local de trabajo y adaptación al mercado y geometría cambiante de las redes interempresariales con proveedores y clientes)

Entre los diversos intentos de establecer tipologías de sistemas territoriales de producción una de las más significativas es la propuesta por Mailat y Kebir (1998). la que clasifica estos sistemas en función de dos criterios complementarios, reflejados en el gráfico número 1. Estos criterios son:

- La existencia o no de relaciones de intercambio con el entorno próximo.

\section{Gráfico n¹: Tipos de sistemas territoriales de producción}

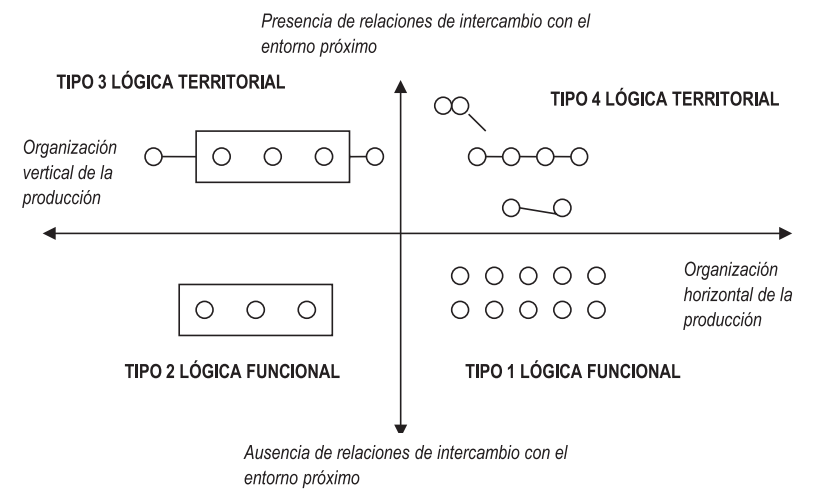

(Fuente: elaboración propia a partir de la información de Mailat y Kebir, 1998). 
- Organización de la producción (vertical u horizontal).

Según este doble criterio, pueden diferenciarse cuatro tipos básicos de situaciones, que pueden caracterizar la actividad productiva de un territorio propuesto por Mailat y Kebir (1998).

Tipo 1: Organización productiva horizontal, sin apenas intercambios con el entorno.

Este tipo de sistema territorial de producción está formado por empresas, tanto grandes como pequeñas, que actúan de manera independiente, generando muy pocos vínculos entre ellas pese a su proximidad, lo que no favorece el aprendizaje interactivo ni la creación de recursos específicos al territorio, que tan sólo actúa como soporte para su actividad. Esas empresas se vinculan, sobre todo, con proveedores y clientes del exterior, lo que las hace muy dependientes en su evolución de circunstancias y decisiones ajenas al propio territorio.

Tipo 2: Organización productiva vertical. sin apenas intercambios con el entorno.

Este segundo tipo se identifica con aquellos casos en que predominan grandes empresas-red, que internalizan la mayor parte de sus actividades, pero segmentándolas a menudo entre diversos establecimientos especializados. Su vinculación, en cambio, a otras empresas localizadas en el entorno resulta muy restringida, generando en consecuencia muy escasos efectos multiplicadores y difusión de conocimientos sobre su entorno, tal como corresponde a verdaderos enclaves aislados de lo que les rodea. En estas condiciones, los recursos generados con la instalación de las empresas desaparecen con ellas si deciden cerrar o trasladarse.

Tipo 3: Organización productiva vertical y relaciones de intercambio con el entorno.

Esta forma de organización se corresponde con la presencia de algunas grandes empresas integradas, con uno o varios establecimientos en la región, pero que a sus propias redes de relaciones internas añaden la formación de vínculos con otras empresas existentes en el entorno, hacia las que externalizan tareas, lo que provoca una densificación de flujos tangibles e intangibles, generalmente de tipo vertical-jerárquico (empresas auxiliares, subcontratadas...). Esto supone una mayor difusión de conocimientos y tecnología desde la gran empresa hacia el resto del sistema, lo que incrementa el potencial de innovación de ese territorio, aunque se mantiene una fuerte dependencia de las decisiones estratégicas tomadas por las grandes firmas.

Tipo 4: Organización productiva horizontal y relaciones de intercambio con el entorno.

Se trata de sistemas territoriales de producción dominados por la presencia de PYMES vinculadas a una o varias cadenas productivas, en donde se genera una compleja división del trabajo interfirmas sin una clara jerarquización entre ellas, asociada al establecimiento de todo un conjunto de reglas -muchas veces no escritas- que exigen alternar la competencia con la cooperación, así como un cierto grado de confianza recíproca.

La coincidencia de algunas de las características señaladas con las que son propias de lo que a continuación se identifica como medios innovadores ha llevado a algunos estudiosos como Storner (1993), a utilizar el término distrito tecnológico, para hacer referencia a este tipo de espacio emergente. Por su parte, Pecqueur (1989) precisa más considerando a los distritos tecnológicos como la segunda generación de sistemas industriales locales. Dada la importancia adquirida por la innovación como factor que propicia la competitividad y el desarrollo, interesa ahora sobre 
todo profundizar en el conocimiento de los medios innovadores, para intentar conocer cómo pueden evolucionar los sistemas productivos locales hacia la conformación de este tipo de medios.

\subsubsection{El territorio como protagonista de} la innovación: los medios o entornos innovadores.

Se han realizado interpretaciones teóricas sobre el dinamismo socioeconómico de ciertos ámbitos que, basando su competitividad en la existencia de redes empresariales y en su capacidad para incorporar conocimientos, se integran con éxito en un sistema económico cada vez más globalizado, pudiéndoseles considerar por ello como espacios emergentes (Caravaca, 1998). Con tales planteamientos se han desarrollado toda una serie de investigaciones sobre distritos industriales (Becatini 1979; Sforzi, 2002; Bagnasco, 2003...) sistemas productivos locales (Pecqueur,1989; Garofoli, 1992...) y medios innovadores (Aydalot, 1986; Camagni, 1989; Maillat, 1993...) que han servido de base para interpretar procesos de desarrollo local y para implementar políticas públicas.

Se puede definir un medio innovador como un conjunto de relaciones que intervienen en un área geográfica que reagrupa, en un todo coherente, un sistema de producción, una cultura técnica y unos actores. El espíritu de empresa, las prácticas organizativas, los comportamientos empresariales, la forma de utilizar las técnicas, de aprehender el mercado y el saber hacer son, a la vez, partes integrantes y partes constitutivas del medio (MaillatQuévit,.-Senn, 1993, 4).

Los medios innovadores han sido objeto de atención por parte de otros investigadores, que han incorporado un enfoque ecológico a los estudios de economía y geografía industrial, interesándose por definir las condiciones territoriales que resultan más favorables para el surgimiento de iniciativas, la formación de redes empresariales y la generación de innovaciones, ya se apliquen a los procesos, los productos o la gestión y organización interna de las firmas, frente a la opción de tomar como punto de partida la empresa o la propia innovación tecnológica.

De forma resumida, se puede indicar que un medio innovador, se compone de una serie de elementos interrelacionados de forma sistémica, pero que pueden ser analizados de forma autónoma, por representar otras tantas dimensiones de esa realidad:

- Un substrato territorial de ámbito local.

- Un conjunto de actores (empresas, instituciones públicas, sindicatos y asociaciones, centros educativos y de investigación...) con capacidad de decisión.

- Una serie de recursos materiales (establecimientos industriales y de servicios, infraestructuras técnicas, patrimonio urbano...) e inmateriales (saber hacer, herencia cultural...), que son comunes y que se intentan identificar y poner en valor.

- Una lógica de interacción, por la que se establecen relaciones entre los actores y existe cierta capacidad de llegar a acuerdos, lo que permite alcanzar las llamadas economías de convención.

- Una lógica de aprendizaje, o capacidad de los actores para modificar su comportamiento a lo largo del tiempo con objeto de adaptarlo de forma flexible a los cambios del entorno.

En coincidencia con lo señalado respecto a los sistemas productivos locales, la 
investigación sobre medios innovadores ha llamado la atención sobre el hecho de queel territorio no actúa como simple escenario inerte y neutral donde se localizan las empresas y desarrollan sus procesos de innovación, sino que interactúa con ellas, favoreciendo o dificultando su avance, al tiempo que orienta la evolución seguida en una determinada dirección o trayectoria, lo que da lugar a procesos acumulativos.

El intento de comprender por qué ciertos territorios evolucionan hasta constituirse en medios innovadores, mientras que otros muchos lo hacen en otras direcciones completamente distintas, puede alcanzarse de forma más eficaz mediante el uso de un esquema argumental inspirado en los enfoques realistas. De este modo, pueden combinarse dos tipos de factores complementarios:

- Condiciones previas territoriales (económicas, sociales, laborales, históricas, culturales...) que propician el surgimiento y rápida difusión de las innovaciones.
- Existencia de agentes locales -privados, públicos o ambos en combinacióncapaces de poner efectivamente en valor esas precondiciones favorables y animar el proceso en una dirección económicamente viable.

La figura 2 recoge una interpretación de Maillat et al. (1993) sobre los diferentes tipos de territorios que pueden surgir según existan o no redes empresariales locales y según la capacidad innovadora de las empresas, las instituciones y la sociedad en su conjunto, concluyendo que son aquéllos en los que las redes están presentes, distritos industriales y medios innovadores, los que se comportan de forma más dinámica y competitiva, por lo que han sido considerados como emergentes.

Maillat et al. (1993) consideran el medio innovador como un sistema complejo en continua evolución, aunque predomine en ellos el componente endógeno, los medios innovadores no se comportan nunca como sistemas cerrados, sino que la innovación

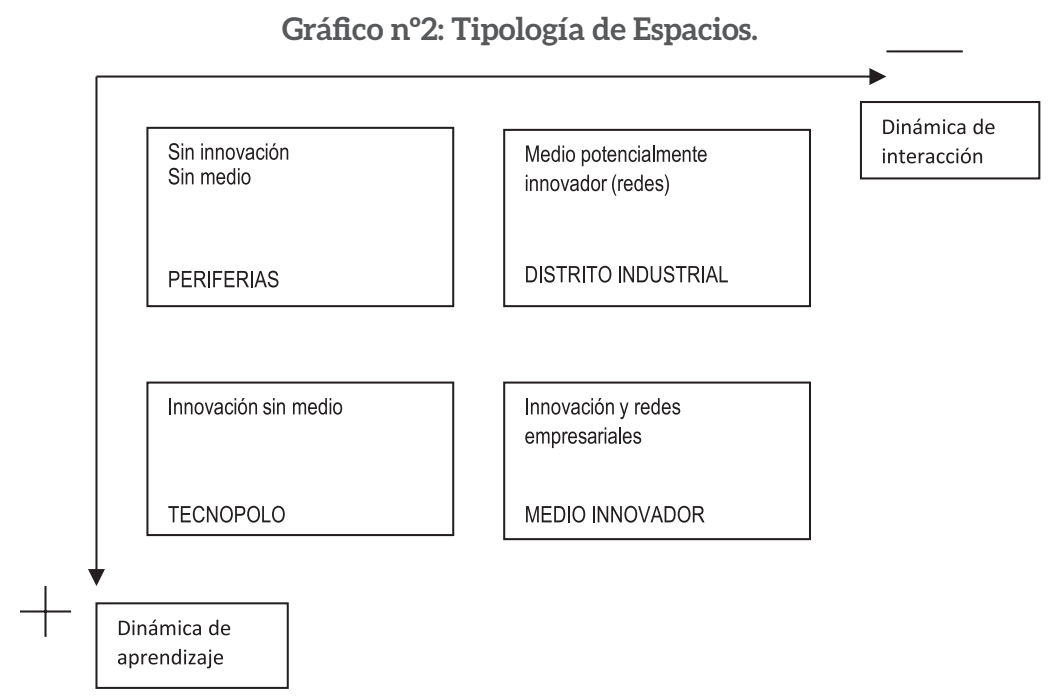

(Fuente: Elaboración propia a partir de la información de Maillat, 1993). 
suele acelerarse y profundizarse en aquellos más abiertos al exterior, de donde pueden proceder:

- Información y conocimientos técnicos.

- Tecnología incorporada en maquinaria y equipos, o no incorporada (bajo la forma de patentes, servicios de asistencia técnica, etc.)

- Recursos económicos y humanos cualificados.

- Demandas de mayor calidad por parte de los clientes, que inducen innovaciones en los proveedores.

\subsubsection{Las redes de cooperación como prin- cipio generador de medios innovadores}

En este estudio interesa la perspectiva reticular, en la que la red es un mecanismo integrador y generador de innovaciones que facilita la comprensión de la compleja dinámica espacial. La red acelera los procesos de difusión tanto de la innovación como de la tecnología, ya que permite el acceso al «aprendizaje» tecnológico de las empresas en su conjunto. El trabajo de Powell et al. (1996) faculta el salto del concepto de learning organization al de learning network. El desarrollo siguiente lleva al aprendizaje colectivo -collective learning-, (Lawson y Lorenz, 1999). El origen del aprendizaje colectivo está en los estudios de los factores determinantes del proceso de innovación y de la competitividad observada en ciertos clusters de actividades de alta tecnología (Keeble y Wilkinson, 1999).

Si se tiene en cuenta que la innovación es una actividad colectiva, resultado de la acción de un conjunto de actores heterogéneos que establecen un sistema de relaciones basadas en la cooperación. Se puede entender entonces que, los verdaderos medios innovadores muestran una organización interna de tipo reticular, resultado de la interacción frecuente y flexible entre los múltiples agentes implicados -en especial las empresas- a la que se identifica habitualmente como red de innovación. Esas redes de innovación se definen como tipos de organización orientados a la innovación, que asocian varias organizaciones jerárquicas (empresas). Suponen, pues, la existencia de un espacio complejo y articulado, en el que existe una elevada densidad de vínculos, tanto entre la mayor parte de las empresas implantadas, como entre éstas y su entorno social e institucional (administración regional y local, universidades, centros de I+D, laboratorios...), que son tanto materiales como inmateriales, destacando por encima de todos los que se relacionan con la información. Como resultado de tales interrelaciones de carácter estable -aunque cambiantes en el tiempo- se genera un valor añadido que beneficia a todos los participantes y que no puede aparecer allí donde las empresas trabajan aisladas y poco conectadas a su entorno local.

Según indica Maillat et al. (1993), identificar un medio innovador supone, por tanto, comprobar la existencia de una verdadera red de innovación y caracterizar sus rasgos principales, que pueden resultar muy variables en función del propio medio en que surgen y de su historia. Esas diferencias se reflejarán en aspectos tales como:

- La dimensión o número de participantes en la red.

- La densidad de los flujos y el mayor o menor grado de conectividad de sus componentes.

- La geometría de los flujos, que pueden ser principalmente verticales/ jerárquicos allí donde unas cuantas empresas dominan la red, $\mathrm{u}$ horizontales en caso contrario. 
- La extensión territorial de los flujos, que pueden desbordar o no el ámbito local.

- La intensidad de sus relaciones con el exterior y su consiguiente posición dentro de la división espacial del trabajo.

En el funcionamiento de esas redes de innovación, la cooperación se constituye en uno de sus motores principales y, por tanto, resulta también un claro elemento de diferenciación respecto a otro tipo de espacios en donde la presencia de empresas innovadoras no se traduce en la creación de un verdadero medio innovador.

\section{METODOLOGÍA}

Los dos aspectos principales en esta investigación serían: la caracterización del sector y la relación de la Universidad de Cádiz con las empresas del sector aeronáutico como sistema de innovación del territorio de la provincia.

El primer aspecto implica realizar una caracterización del sector. Para ello se realiza un breve diagnóstico previo del territorio en estudio. Mediante el análisis de la bibliografía disponible se pretende caracterizar la evolución del sector aeronáutico en Andalucía y Cádiz en los primeros años del s. XXI.

Se determina el periodo de 2002 a 2011 como el idóneo para este estudio debido a la existencia de datos de multitud de variables, y a que en este período se producen el inicio de las colaboraciones de la Universidad de Cádiz y el sector en la provincia de Cádiz.

Se han seleccionado los datos más relevantes que permiten caracterizar el impacto de esta colaboración y el papel de la Universidad como agente dinamizador del desarrollo en el territorio de la provincia de Cádiz. Destacar las variables de empleados y facturación anuales en el sector, en Andalucía y en la provincia. Del mismo modo también se analiza el espectro de las empresas existentes.

El segundo aspecto consiste en determinar la relación de la Universidad de Cádiz con las empresas del sector aeronáutico como sistema de innovación del territorio de la provincia. Para ello se realiza una búsqueda bibliográfica y posterior selección de la información considerada más idónea para esta caracterización.

\section{ANÁLISIS}

\subsection{Caracterización del sector}

En esta fase de la investigación, se profundiza en el sector aeronáutico, en primer lugar se procede a exponer brevemente la evolución del sector en España, posteriormente se procede a caracterizar el sector su evolución e influencia en el territorio andaluz y enfatizando lo posible en los datos relativos a la provincia de Cádiz, focalizada tal influencia en los términos de Puerto Real y El Puerto de Santa María. La justificación de esta elección, es el establecimiento en ambas ciudades de las dos empresas tractoras principales que tiene el sector. Airbus Defense and Space en El Puerto de Santa María y Airbus Operations en Puerto Real.

Se pretende caracterizar la evolución del sector para poder tener una imagen completa que permita poder determinar el alcance del impacto sobre el desarrollo Territorial.

\subsubsection{El sector aeronáutico en España}

Para caracterizar el sector se emplean los datos recogidos en los informes de la 
agencia IDEA del sector aeronáutico del año 2011 y el informe ejecutivo resumen del año 2011 de la fundación hélice y los datos disponibles acerca de la historia del sector disponible en la página web: "www. pasionporvolar.com".

Hablar de origen del sector, obliga a introducir la empresa tractora por excelencia del sector en España que ha dinamizado y creado el tejido empresarial del sector. Se trata de la empresa Construcciones Aeronáuticas S.A. (CASA) fue creada por José Ortiz Echagüe el 3 de marzo de 1923. Construcciones Aeronáuticas S.A.(CASA)iniciósustrabajos en mayo de 1924 en que se inauguró la factoría de Getafe, construyendo bajo licencia aviones Breguet. En 1926 se construyó en Cádiz una segunda factoría, para la construcción bajo licencia del hidroavión Dornier Do J Wal.

Terminada la guerra civil, CASA abrió una nueva factoría en Tablada, Sevilla, tras conseguir la fabricación bajo licencia de algunos modelos alemanes. A partir de 1943 el Estado Español invierte en esta compañía. Empezó participando con un $33 \%$, aunque en 1992 ya controlaba el $99,2 \%$ de la empresa. En 1945 inaugura su fábrica en Madrid, dedicada al mecanizado. En 1946 se restablece la oficina de proyectos. De esta manera se reanuda el diseño de aviones propios con tecnología propia. Este es un punto importante a tener en cuenta, ya que una serie de problemas había convertido a la compañía en un fabricante de aviones con licencias extranjeras, pero sin tecnología para la fabricación de proyectos propios.

En 1971 se autoriza la fusión por absorción de Hispano Aviación, S.A.. En 1972, CASA pasa a ser miembro del Grupo de Interés Económico Airbus. En 2000, CASA se integra en el grupo EADS y pasa a denominarse EADS CASA.

Construcciones Aeronáuticas (CASA), en el año 1999 es comprada por Daimler y por Aeroespatiale, y se forma como un triunvirato, que posteriormente sirve para formar EADS en diciembre de 1999 esa estructura que es la que hoy día aún se mantiene. Posteriormente entre EADS y BAE Systems (poniendo BAE un 20\% y EADS el 80\%) se crea Airbus España; pero a la antigua CASA se divide en dos, en lo que se llama EADS CASA y por el otro lado aparece Airbus España, al igual que aparecerán otra serie de Airbus en varios países. En EADS CASA se fabrican aviones de transporte militar, aviones militares, $y$ espacio, conocido por las estructuras que se hacen para Arianne, los satélites, etc.

Tomando como referencia el dato del año 2011, Andalucía se consolida como la segunda región de España en el sector aeronáutico, lo que la convierte a su vez en una de las principales regiones de Europa en este sector. Como se puede ver en el gráfico número 3, la cifra de empleo de la Comunidad andaluza representa un 29\% del total nacional, con un número de empleados en el sector que casi duplica a la tercera región española.

\section{Gráfico n³. Empleo en el sector aeronáutico en España.}

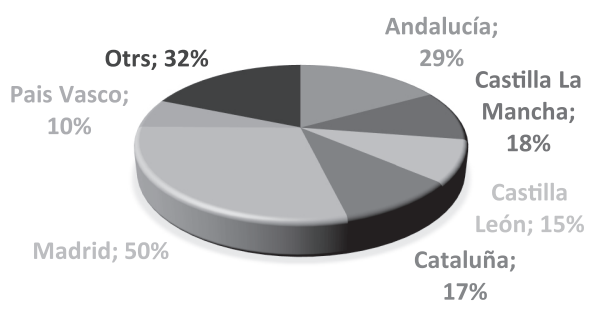

(Fuente: Elaboración propia a partir de informe anual 2011 fundación hélice).

Asimismo, el volumen de ventas registrado por comunidades autónomas en 2011, 
es nuevamente un claro indicador de la posición predominante del sector a nivel nacional que está ocupando Andalucía, así lo indican los 1754 millones de euros facturados el 2011, que representan un $24 \%$ del total en España, esto se ve reflejado en el gráfico número 4.

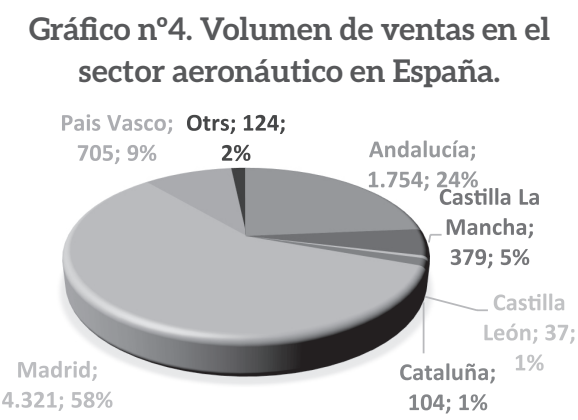

(Fuente: Elaboración propia a partir de informe anual 2011 fundación hélice)

\subsubsection{Caracterización del Sector aeronáu- tico en el contexto de Andalucía.}

Para caracterizar el sector se emplean los datos recogidos en los informes de la agencia IDEA del sector aeronáutico del año 2011 y el informe ejecutivo resumen del año 2011 de la fundación hélice.

Se comienza la caracterización por las grandes empresas tractoras, las cuales han sido motor del tejido empresarial auxiliar y el sistema de gestión de la innovación creado en el territorio andaluz alrededor de este sector. EADS-CASA y Airbus son las dos principales empresas aeronáuticas con presencia en Andalucía. Las plantas de desarrollo y ensamblaje de estas compañías en la región participan en importantes proyectos internacionales, posicionándose como centros de vanguardia en sus respectivas especialidades. EADS-CASA está presente principalmente en la provincia de Sevilla y en menor medida en Cádiz, mientras que Airbus está ubicada en Cádiz.
- Centro de EADS CASA en el Tecnoparque Bahía de Cádiz (Puerto de Santa María)

Destaca por la tecnología empleada en fabricación de estructuras y componentes de fibra de carbono, en tecnología de conformados superplásticos y en chapistería integral.

- Planta de Airbus España en Puerto Real (Cádiz)

Esta planta está dedicada al montaje de aeroestructuras en materiales metálicos y fibra de carbono para la división de aviación civil para los estabilizadores de la familia Airbus.

- Planta de San Pablo (Sevilla) de EADSCASA

En esta planta se realizan actividades de ensamblaje y equipado final de los aviones militares C212, C295 y C235 y del futuro A400M. Además es centro de mantenimiento de aeronaves.

- Fábrica de Tablada (Sevilla)

Esta fábrica pertenece también a EADSCASA. Se dedica fundamentalmente al control numérico de mecanizados de alta velocidad, conformado y fresado químico de revestimientos, así como al montaje de aeroestructuras.

- Centro de ensamblaje del A400M en San Pablo (Sevilla)

Es la planta en la que se realiza el ensamblaje final, pruebas en vuelo y entrega del avión A400M.

La evolución positiva del sector andaluz viene siendo una realidad desde 2002.

En este periodo, el sector aeronáutico andaluz ha experimentado en la última década un avance sin antecedentes en ninguna otra región española, con un crecimiento del $244 \%$ de la facturación (1.246 Millones de euros) y del $279 \%$ del número de empleos (6935 empleos más). 
La evolución desde 2002 hasta 2011 respecto a la facturación en el sector aeroespacial en Andalucía es ascendente salvo el año 2011 que observa un retroceso, no obstante el crecimiento en general que ha tenido en la primera década del siglo XXI. Se puede observar la evolución en la gráfica número 5 elaborada a partir de los datos del informe de la fundación hélice.
En la gráfico número 6 se puede observar la evolución del empleo en el sector aeronáutico en Andalucía (2002-2011), en este caso el crecimiento es evidente y mantenido en el tiempo.

Es decir, en una década se ha multiplicado por 3 la facturación y el empleo. Lo que ratificaría la gran apuesta que debemos

Gráfico ${ }^{\circ}$ 5. Distribución de la facturación del sector aeronáutico en Andalucía en la última década.

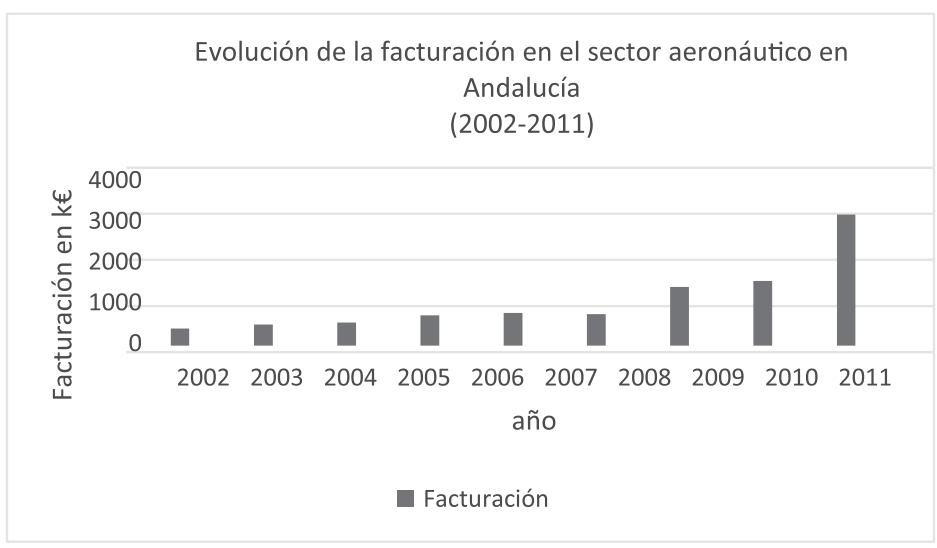

(Fuente: elaboración propia a partir del informe del año 2011 fundación hélice)

\section{Gráfico $n^{\circ}$ 6. Distribución del empleo y facturación del sector aeronáutico en Andalucía en la última década.}

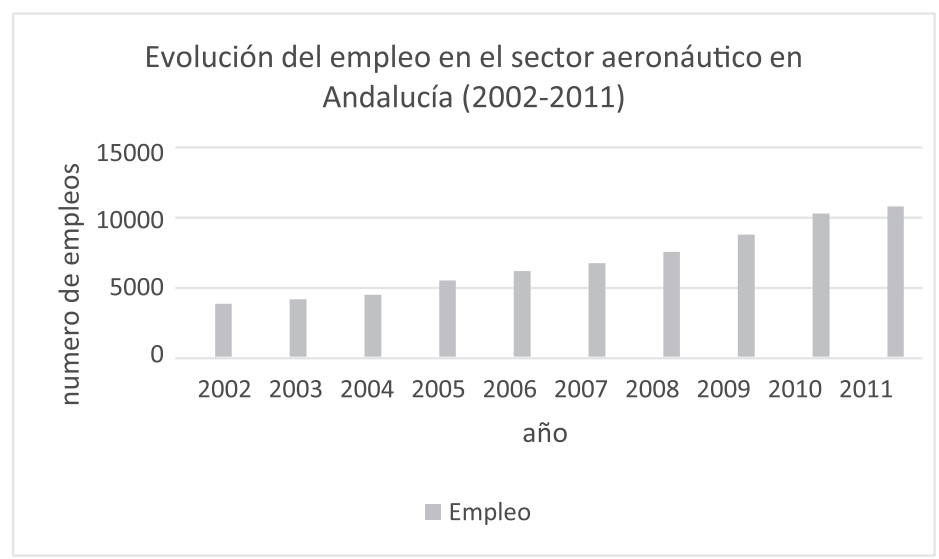

(Fuente: elaboración propia a partir del informe del año 2011 fundación hélice) 
hacer alrededor del desarrollo del sector y las oportunidades de negocio que se siguen creando año tras año. En la tabla número 1 se puede observar esta evolución referido a facturación con todos los datos entre 2002 y 2011.

\section{Tabla n¹. Distribución del empleo y facturación del sector aeronáutico en Andalucía en la última década.}

\begin{tabular}{|c|c|c|}
\hline Año & Facturación $(\mathbf{m} €)$ & $\mathbf{N}^{\circ}$ Empleados \\
\hline 2002 & 509 & 3.867 \\
\hline 2003 & 596 & 4.179 \\
\hline 2004 & 645 & 4.516 \\
\hline 2005 & 799 & 5.535 \\
\hline 2006 & 848 & 6.206 \\
\hline 2007 & 825 & 6.753 \\
\hline 2008 & 1.417 & 7.555 \\
\hline 2009 & 1.541 & 8.786 \\
\hline 2010 & 2.983 & 10.278 \\
\hline 2011 & 1.754 & 10.802 \\
\hline
\end{tabular}

(Fuente: Elaboración propia a partir de informe anual 2011 fundación hélice)

Según el instituto hélice en su informe ejecutivo del año 2011. El eje Sevilla-Cádiz aglomera al 90\% de las empresas del sector, siendo este porcentaje aún mayor si el mismo se calculase en función del empleo soportado o la facturación aportada desde los centros de trabajos.

En concreto en Cádiz se concentran el 19\% de las empresas andaluzas que totalizan 23 empresas. De estas 23 empresas, se puede destacar las siguientes: AIRBUS OPERATIONS., AIRBUS D\&S, ALESTIS AEROSPACE, AERNNOVA, CARBURES, TITANIA, AEROTECNIC, AEROSERV, LTK, KUENE NAGHEL

Se observa que en esta última década se ha producido un aumento del $271 \%$ en el empleo de la provincia de Cádiz. Es decir, prácticamente se ha triplicado el empleo.
Esta evolución se puede observar en la tabla número 2 .

\section{Tabla n². Distribución del empleo del sector aeronáutico en Andalucía en la última década.}

\begin{tabular}{|c|c|c|}
\hline Año & $\begin{array}{c}\text { Sevilla. Número } \\
\text { de empleados }\end{array}$ & $\begin{array}{c}\text { Cádiz. Número de } \\
\text { empleados }\end{array}$ \\
\hline 2002 & 2.914 & 951 \\
\hline 2003 & 3.065 & 1.111 \\
\hline 2004 & 3.189 & 1.322 \\
\hline 2005 & 4.170 & 1.327 \\
\hline 2006 & 4.404 & 1.703 \\
\hline 2007 & 4.739 & 1.795 \\
\hline 2008 & 5.387 & 1.959 \\
\hline 2009 & 5.970 & 2.349 \\
\hline 2010 & 7.099 & 2.546 \\
\hline 2011 & 7.491 & 2.585 \\
\hline
\end{tabular}

(Fuente: Elaboración propia a partir de informe anual 2011 fundación hélice)

No obstante observar que entre 2010 y 2011 en la provincia de Cádiz, no ha existido un aumento significativo del empleo, no es un dato alarmante debido a que si se estudian la situación de los proyectos del sector se observa que el año 2010 y 2011 son años de desarrollo de los nuevos programas que están a punto de entrar en serie como son el A350 WXB cuyo efecto principal sería sobre las empresas AIRBUS, ALESTIS y AERNNOVA y el A400M cuyo impacto principal sería para las empresas AIRBUS MILITARY y CARBURES. Las empresas del sector más importantes en la provincia de Cádiz serían AIRBUS OPERATIOS, AIRBUS DEFENSE \& SPACE, ALESTIS, en menor medida se puede citar a LTK y AEROSERV.

Según el instituto hélice en su informe ejecutivo del año 2011, podemos observar en la siguiente figura número 7 la distribución por actividad de las industrias aeronáuticas andaluzas. 
Gráfico $n^{\circ}$ 7. Distribución del tipo de empresas del sector establecidas en

Andalucía en el año 2011.

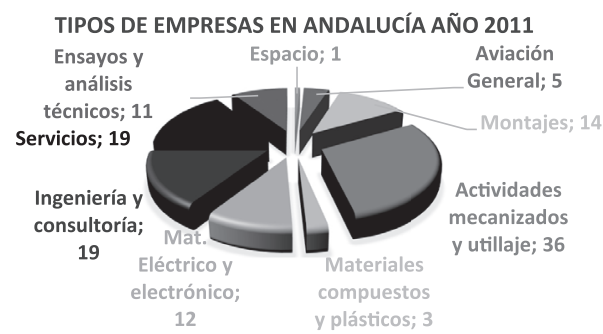

(Fuente: Elaboración propia a partir de informe anual 2011 fundación hélice)

En el caso de la provincia de Cádiz:

- Montajes. ALESTIS, AIRBUS OPERA-TIONS, AIRBUS D\&S, AERNNOVA

- Actividades mecánicas y utillajes: AEROTECNIC, INFASUR.

- Servicios:LTK, AEROSERV, KUENENAGHEL
Según el instituto hélice en su informe ejecutivo del año 2011, se ha realizado la siguiente gráfica muestra la evolución en lo últimos años del empleo del sector en Andalucía, en el que se puede observar la importancia creciente del tejido industrial auxiliar. Y como se ha comentado en los hitos anteriores el crecimiento espectacular desde el año 2003 en el tejido auxiliar aeronáutico.se puede observar en el gráfico número 8.

Igualmente el crecimiento de la facturación de las empresas auxiliares se puede observar en el gráfico número 9, algo lógico tras ver el aumento del empleo. Y es un dato del desarrollo que ha tenido el sector auxiliar en la comunidad y en concreto en alrededor de las empresas tractoras del eje Sevilla-Cádiz.

\section{Gráfico $n^{\circ}$ 8. Distribución del empleo del sector aeronáutico en Andalucía en} la última década en las empresas auxiliares y tractores.

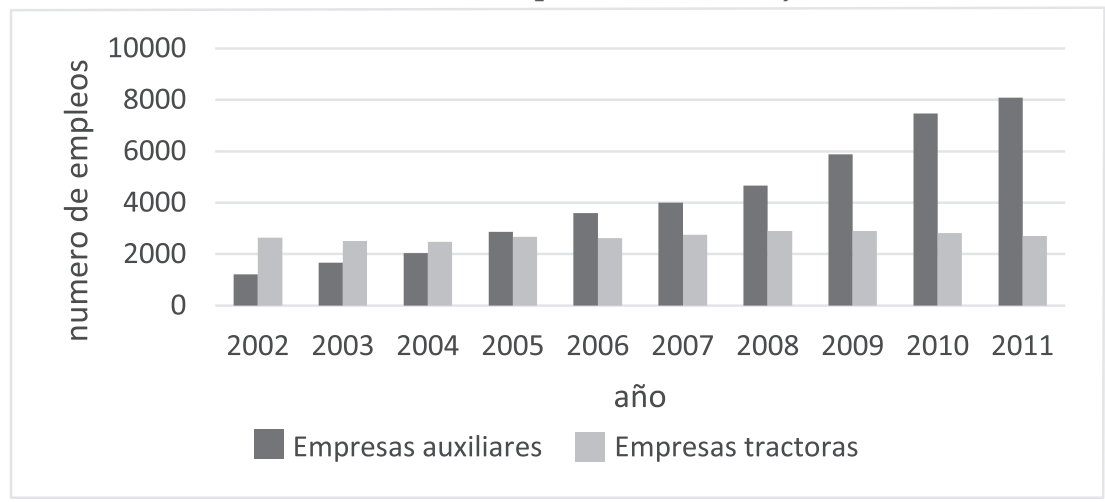

(Fuente: elaboración propia a partir de datos del informe anual 2011 fundación hélice)

4.2. La relación de la Universidad de Cádiz con las empresas del sector aeronáutico como sistema de innovación del territorio de la provincia.

\subsubsection{Aspectos Generales.}

Las evidencias empíricas y los enfoques teóricos más recientes de la economía regional ligados a los conceptos de "medio innovador", "distrito industrial" o "sistema productivo local", ponen de manifiesto que el desarrollo regional, en un entorno de creciente competitividad en los mercados, depende de la capacidad de propiciar la innovación en áreas geográficas concretas.

Estas áreas innovadoras son, principalmente, las regiones urbanas o tecnópolis y 
Grafico $n^{\circ}$ 9. Distribución de la facturación del sector aeronáutico en Andalucía en la última década en las empresas auxiliares y tractores.

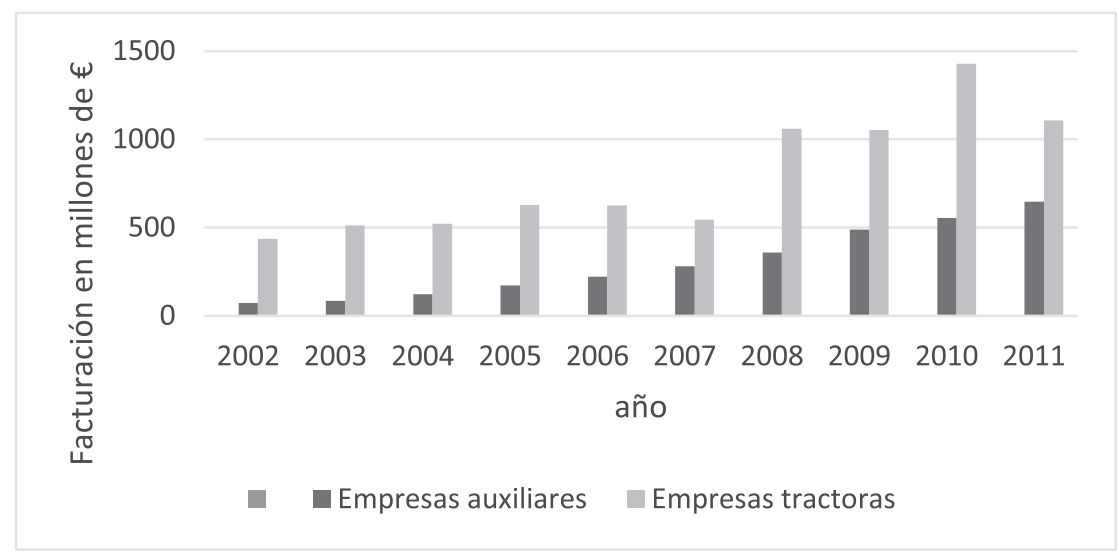

(Fuente: elaboración propia a partir de datos del informe anual 2011 fundación hélice)

parques tecnológicos centrados en industrias de alto nivel tecnológico, actividades de I+D y servicios avanzados; pero también y de forma creciente determinados sistemas productivos locales.

Éstos son municipios en los que se ha conformado un clima empresarial, social, cultural e institucional favorable y que albergan concentraciones de pequeñas y medianas empresas (PYMES) especializadas en una determinada actividad económica vinculada habitualmente a una tradición artesanal o industrial preexistente. Insertada en una lógica de competencia y cooperación simultánea, estas empresas se encuentran articuladas entre sí por lazos comerciales y redes de aprendizaje y cooperación empresarial y todas ellas se benefician de la proximidad geográfica en forma de diferentes externalidades positivas y efectos sinérgicos.

Andalucía no es ajena a estas tendencias internacionales y participa plenamente del fenómeno de los sistemas productivos locales. Como consecuencia y, a su vez, expresión del profundo proceso de modernización de nuestra Comunidad Autónoma durante las últimas dos décadas, existen en Andalucía varias áreas en las que en torno a diferentes actividades industriales se han formado y se están consolidando en la actualidad sistemas productivos locales.

La aportación de estos sistemas productivos locales al progreso económico y social de nuestra Comunidad Autónoma no puede ser infravalorada, ya que estos sistemas productivos locales fortalecen el tejido empresarial, generan empleo y renta, y coadyuvan, en la medida en que son capaces de generar o, al menos, incorporar innovaciones, a la mejora de la competitividad de Andalucía. Asimismo, ha de destacarse que estos sistemas productivos locales fomentan la cohesión económica y social de sus respectivos territorios de influencia y, con su lógica de cooperación, contribuyen también al incremento del capital social de Andalucía.

En el caso que nos ocupa, la Agencia de Innovación y Desarrollo de Andalucía IDEA, en su objetivo de promover el 
desarrollo del sector aeronáutico en Andalucía, cuenta con una dirección especializada en este sector estratégico. Asimismo, ha impulsado la puesta en marcha de centros tecnológicos y organismos de apoyo a esta industria en Andalucía.

En lo que respecta a la provincia de Cádiz dispone de un Parque Tecnológico. El Parque Tecnológico denominado TecnoBahía, es un conjunto de infraestructuras y servicios tecnológicos ubicados en la Bahía de Cádiz, promovido por la Agencia de Innovación y Desarrollo de Andalucía, agencia de desarrollo regional del Gobierno Andaluz adscrita a la Consejería de Economía, Innovación y Ciencia.

Tiene como misión el desarrollo de la dotación tecnológica de sus empresas objeto y del resto del sector industrial de la provincia de Cádiz, y en especial de la Bahía de Cádiz, propiciar la creación de Empresas de Base Tecnológica y la transformación de las existentes en este tipo de empresas y promocionar y dinamizar la innovación, el desarrollo tecnológico y la transferencia de tecnología. El Parque Tecnológico Bahía de Cádiz, Tecnobahía se asienta en la provincia de Cádiz, en tres plataformas complementarias, una en el Puerto de Santa María (Parque Industrial Bahía de Cádiz y dos en Puerto Real (Parque Industrial El Trocadero y Parque Industrial Las Aletas). Está calificado como Parque Científico-Tecnológico por la Consejería de Economía, Innovación y Ciencia. Tecnobahía coordina los esfuerzos de estos tres parques industriales, además de un centro de incubación y servicios avanzados para empresas de la industria de nuevos materiales, prestando especial atención a sus aplicaciones para el sector aeronáutico, naval y de la automoción. Su misión se centra en promover el espíritu de la innovación en estas indus- trias, la creación de Empresas de Base Tecnológica y la incorporación de sistemas y tecnologías de vanguardia que mejoren su competitividad. Su entidad gestora, Tecno Bahía S.L., está participada por la Agencia de Innovación y Desarrollo de Andalucía IDEA, y por la Universidad de Cádiz, y con ella colaboran la Red Transfronteriza de Servicios a las Empresas (RETSE) y la Red de Espacios Tecnológicos de Andalucía (RETA) ofreciendo, en conjunto, un amplio abanico de servicios tecnológicos a las empresas y entidades de su ámbito de actuación.

\subsubsection{El papel de la Universidad de Cádiz como dinamizador.}

Mediante el análisis de las publicaciones y noticias se pueden establecer las características de la Universidad de Cádiz comodinamizador deldesarrolloterritorial provincial en el sector aeronáutico. Para ello destacaremos el proyecto inicial y posteriormente se resumirán la situación posterior a 2008 hasta la actualidad en base a las diferentes iniciativas realizadas y los datos obtenidos.

\subsubsection{Proyecto inicial: Primera fase de Colaboración con Airbus. 2003-2008}

El papel de la Universidad de Cádiz es fundamental en este sistema de desarrollo territorial, como se ha comentado forma parte de la entidad gestora del parque. Esa conexión entre el sector y la Universidad puede considerarse que tiene su origen en el proyecto de colaboración iniciado hace más de una década entre varios departamentos de la Universidad de Cádiz y la planta de Puerto Real de Airbus Operations.

En concreto en 2008, se realiza un estudio para presentar un dossier para la primera 
edición los premios "implicación social en las Universidades", para reconocer las mejores prácticas de colaboración entre las universidades públicas de Andalucía y las empresas y otros organismos públicos y privados de su entorno.

El Consejo Social de la Universidad de Cádiz propuso para este premio a la planta de Puerto Real de Airbus España, uno de los principales proveedores mundiales de aviones comerciales, dada la colaboración existente entre la Universidad de Cádiz y esta empresa, con planta en Puerto Real. Una colaboración que abarca prácticas en empresa, trabajos de fin de carrera, de investigación, colaboraciones como profesores asociados $\mathrm{y}$ de master $\mathrm{y}$ postgrados, visitas de alumnos, y feria de empleo, entre otras actividades y acciones. Del documento publicado en su momento que se puede consultar en la referencia web incluida en la bibliografía referida al consejo de social de Andalucía, de tal documento se extraen los siguientes puntos claves.

La idea principal de esta colaboración es potenciar que los Grupos de Investigación trabajen coordinadamente con la industria con el fin de fijar objetivos comunes y garantizar que esta transferencia incremente todavía más el valor añadido para el sistema empresarial.

La relación entre la Empresa AIRBUS y la Universidad de Cádiz parte del año 2000 donde se inician los primeros contactos $\mathrm{y}$ se ponen en marcha los primeros proyectos. La relación fundamentalmente del Departamento de Ingeniería Química inicia el desarrollo de trabajos comunes. Así se firma el primer convenio Universidad y la Empresa en el año 2001, los proyectos fin de carrera el año 2000 y el proceso de prácticas en Empresa ya se consolida en el año 2002, manteniendo una regularidad en el mismo hasta hoy. Se participa en las Ferias de Empleo de la Universidad desde el año 2004. Varios de sus directivos han ejercido como profesores asociados o como profesores en Másteres y Postgrados y siempre la Empresa ha estado dispuesta a la colaboración solicitada por la universidad para presentaciones a grupos de alumnos, bien en centros universitarios o en la propia factoría, o para poner a disposición de la universidad sus instalaciones o personal.

Fundamentalmente los beneficiarios fueron alumnos, profesores, la propia universidad y la empresa, de forma directa e indirectamente el desarrollo socioeconómico de la comarca ya que como resultado de dicha colaboración han participado de la misma Departamentos Universitarios; los servicios universitarios de postgrado y de transferencia de tecnología (OTRI) los Centros de Ciencias y de Ingeniería, los servicios de empleo de la Universidad y sin poder cuantificar los numerosos alumnos que han pasado por las presentaciones realizadas en las Ferias de Empleo, cursos de postgrado y de doctorado, colaboraciones como profesores asociados.

El impacto del proyecto de colaboración es un ejemplo de la necesaria conexión universidad - entorno tanto para la formación, como para la investigación y la innovación para el desarrollo de la zona. Y desde los que se puede ver la importancia que tanto para la empresa, que resuelve problemas con ayuda de la universidad, como para la universidad que le permite transferir conocimiento al entorno en el que se mueve a la vez que conseguir especializar a sus alumnos y titulados en materias vivas y necesarias.

Respecto al alcance del proyecto, comentar que los alumnos a través de su 
participación mediante los proyectos de fin de carrera, fundamentalmente en las especialidades de Ingeniería Química e Ingeniería Industrial, adquieren de forma práctica y real su especialización en temas como: Implantación de las herramientas "lean manufacturing", a distintos procesos de aprovisionamiento y fabricación; gestión del sistema documental de la trazabilidad de elementos Estructurales en la fabricación de estructuras metálicas; diseño, implantación y certificación según UNE-EN-9100 de diferentes procesos industriales, etc

El mejor resultado de esta colaboración fue el nacimiento de la primera Empresa de Base Tecnológica surgida a raíz del programa "Campus" de la Consejería de Innovación, Ciencia y Empresa. En concreto se creó "Easy Industrial Solutions", que posteriormente ha derivado en el grupo CARBURES.

En este proyecto se han involucrado prácticamente todos los Servicios Universitarios: Dirección General de Empleo; OTRI; FUECA; Departamentos y centros universitarios, Vicerrectorado de Alumnos y Vicerrectorado de Investigación. Su grado de implicación ha consistido en la colaboración, el desarrollo y el aprendizaje de tareas.

Los resultados principales de esta colaboración se detallan a continuación:

- 6 Departamentos Universitarios involucrados

- 2 convenios de colaboración y 14 contratos OTRI con un montante de más de $400.000 €$

- 86 proyectos Fin de Carrera

- 153 prácticas en empresa

- 2 Empresas de base tecnológica. (EASY INDUSTRIAL SOLUTIONS \& TITANIA)

\subsubsection{Otros proyectos de colaboración} con el sector aeronáutico. 2003-2014

Como se ha comentado anteriormente el proyecto principal de colaboración de la Universidad de Cádiz respecto al sector es con Airbus, para realizar una adecuada caracterización del papel de la Universidad de Cádiz en el sector aeronáutico de la provincia de Cádiz es necesario recapitular todos los proyectos que la Universidad también ha realizado y que igualmente han contribuido a dinamizador el sector, en este caso a nivel de empresas auxiliares.

\section{- Firma de 5 convenios entre Universidad} de Cádiz y Airbus España. (2013-2014)

\begin{abstract}
Airbus y Universidad de Cádiz han formalizado un marco amplio y estable de colaboración en el desarrollo de actividades de interés mutuo, que permite tejer una sólida relación científica, tecnológica y educativa para abordar proyectos conjuntos de formación, de prácticas en empresa y de innovación y transferencia tecnológica en beneficio del desarrollo socio económico.
\end{abstract}

De este modo, se ha materializado una intensa relación institucional entre la Universidad de Cádiz y Airbus que, ahora, mediante el desarrollo de iniciativas, proyectos y acciones conjuntas, potenciarán la formación de calidad, la internacionalización y la colaboración en la realización de proyectos tecnológicos de interés común.

(Noticia publicada en la web de la Universidad de Cádiz incluida en la bibliografía)

\section{- Proyecto "campus".}

Otro ejemplo claro de dinamización del sector, en este caso no solo facilitando 
la transferencia tecnológica entre universidad y empresas, si no que facilita la creación de iniciativas emprendedoras creadoras de riqueza en el territorio.

o Desarrollo de Carbures, sector de consultoría y composites, a partir de la spin off Easy industrial Solutions.

o Desarrollo de Titania, dentro de los ensayos no destructivos.

(Noticia publicada en la web de la Universidad de Cádiz incluida en la bibliografía)

- Implantación del master en ingeniería de fabricación en el campus de Puerto Real y el grado de ingeniería aeroespacial en el campus de Ingeniería de Puerto Real.

Ante la demanda de capital humano especializado en el sector, la Universidad de Cádiz desarrolló dos acciones para por un lado ampliar la polivalencia de ingenieros y ofrecer al sector ingenieros con capacidades adecuadas. Y por otro lado desarrolla un grado de ingeniería específico para poder dar nuevos ingenieros con la formación adecuada a las empresas del sector en la provincia.

El primer curso en el que se inicia esta ingeniería es el 2011/2012. Publicándose en 2012 en el BOE. En el año 2014 comenzó el master de ingeniería de fabricación. (Información consultada de la página web oficial de la Universidad de Cádiz incluida la bibliografía)

- Firma de acuerdos similares al establecido con Airbus.

Para facilitar la integración de los alumnos en el tejido productivo $\mathrm{y}$ facilitar la transferencia de tecnología que se cree de estos convenios de colaboración a partir de la oficina OTRI y todos los departamentos que están involucrados.

\subsubsection{Nuevos proyectos.}

(2016 - actualidad)

El principal proyecto de futuro que involucra a la Universidad de Cádiz como dinamizador del sector aeronáutico en la provincia de Cádiz, adicional al mantenimiento de los acuerdos ya establecidos sería, el que se recogía en la prensa el pasado 27 de septiembre de 2016.

(Noticia publicada en la web de la Universidad de Cádiz incluida en la bibliografía)

En la que se indicaba que "Universidad de Cádiz Junta, Airbus y Navantia firman el protocolo para el Centro de Innovación en Tecnologías de Fabricación Avanzada", el cual se va a ubicar en la Bahía de Cádiz concretamente en Puerto Real. La Universidad de Cádiz colabora desde hace años en proyectos de I+D con las empresas tractoras integrantes de esta iniciativa conjunta. Según la representación de la Universidad de Cádiz, como institución académica tiene misión como servicio público y el compromiso social por el desarrollo del territorio, donde la innovación debe ser fundamental. Se destaca también el hecho de que con este acuerdo se reconoce a la Universidad de Cádiz como motor de desarrollo socio-económico de la provincia de Cádiz.

El CFA, centro de innovación en procesos y tecnologías, dotado con 25 millones de euros, estará vinculado a los conceptos de fabricación avanzada y de factoría del futuro, con especial interés en el sector aeronáutico y naval. Llevará a cabo proyectos de I+D desarrollados por empresas en colaboración con grupos de investigación. 
Destacar también los datos que se presentaron en 2015 como dato global de todo el impacto de la Universidad en la provincia, no sólo del sector aeronáutico, lo se puede encontrar por los datos que resultan del estudio que personal de la propia universidad presentó en 2015. En concreto, los números que se presentaron y resumen la acción de la Universidad de Cádiz y su proyección hacia la sociedad serían unos 5.300 empleos (que se elevarían a 7.135 con la inclusión de sus 1.820 trabajadores de plantilla: 1.111 PDI y 710 PAS) y más de $414 \mathrm{M} €$ (millones de euros) de volumen de negocio que genera su actividad en términos económicos. La presentación tuvo lugar dentro del Seminario sobre impacto de la Universidad de Cádiz organizado por el Consejo Social de la UNIVERSIDAD DE CÁDIZ. (Noticia publicada en la web de la Universidad de Cádiz incluida en la bibliografía).

\section{CONCLUSIONES}

En definitiva la Universidad de Cádiz es un agente dinamizador de la provincia de Cádiz, con su labor de colaboración con las empresas aeronáuticas a través de la transferencia de conocimiento y tecnología, gracias a la promoción de la innovación, desarrollo e investigación conjunta con el sector aeronáutico.

Recordando a Maillat et al. (1993) se observa que el medio innovador como un sistema complejo en continua evolución, aunque predomine en ellos el componente endógeno, los medios innovadores no se comportan nunca como sistemas cerrados, sino que la innovación suele acelerarse y profundizarse en aquellos más abiertos al exterior, es claro que en el caso del sector aeronáutico en la provincia de Cádiz, se ha conseguido un medio innovador ya que no se trata de un sistema productivo cerrado sino abierto al exterior.
Identificando los elementos de ese medio innovador:

- Un substrato territorial deámbito local. En este caso la provincia y concretamente la zona de la Bahía de Cádiz.

- Un conjunto de actores (empresas, instituciones públicas, sindicatos y asociaciones, centros educativos y de investigación...) con capacidad de decisión.. En este caso: la Universidad, agencia IDEA, parques tecnológicos, empresas tractores y asocianes de empresas auxiliares)

- Una serie de recursos materiales e inmateriales que son comunes y que se intentan identificar y poner en valor. Ejemplo las instalaciones de la universidad y las empresas (acuerdos de colaboración), y como inmaterial todos los proyectos realizados.

- Una lógica de interacción: Todos los acuerdos y convenios establecidos, y los intercambios de conocimiento realizados.

- Una lógica de aprendizaje. Ejemplo, el paso de crear el centro de investigación avanzada de fabricación.

La Universidad de Cádiz ha conseguido contribuir al desarrollo del territorio, como lo demuestran los números de contratos de transferencia de tecnología y de personal universitario contratado por las empresas del sector, igualmente han promovido y desarrollado iniciativas empresariales a partir de emprendedores de la propia comunidad universitaria.

Los números presentados ratifican el papel dinamizador de la Universidad inclusive la firma de los nuevos convenios de futuro. Destacar las siguientes conclusiones al respecto del estudio realizado:

- El sector aeronáutico ha seleccionado la provincia de Cádiz como segundo eje en la región de Andalucía. En base a los 
datos presentados, claramente durante la primera década del siglo XXI, el sector en la provincia ha experimentado una evolución paralela a la del primer eje que es Sevilla, reforzando su papel de segundo eje o polo industrial aeroespacial andaluz.

- Se han creado varias empresas proveedoras en torno a la ampliación de la empresa tractora Airbus, a raíz del desarrollo en la primera década del siglo del proyecto A380, continuando con un desarrollo de un gran proveedor debido al lanzamiento del nuevo proyecto A350. En concreto se observan, la dinamización planteada al inicio del estudio ya que la propia universidad de Cádiz fomenta inclusive la creación de alguno de estos proveedores a través de empresas con base tecnológica surgidas desde la Universidad.

- A raíz del desarrollo de la industria en la primera década del siglo se desarrollan empresas públicas y privadas para proveer de formación adecuada a la mano de obra que demanda el sector. Entre las públicas se ha evidencia el desarrollo fundamental de la relación entre el sector y la Universidad de Cádiz como agente fundamental en la dinamización del territorio. En vista de los volúmenes de colaboraciones, prácticas, transferencias tecnológicos, etc.

Se puede concluir que la Universidad de Cádiz forma parte desde el principio de uno de los polos para la innovación y la transferencia del conocimiento con mayor peso estratégico y proyección para el futuro de la provincia de Cádiz y Andalucía, así como colabora desde hace años en proyectos de I+D con las empresas tractoras del sector aeronáutico.

Prácticas de Empresa, Proyectos de Colaboración, Intercambio de experiencias deben ser los pilares de esta relación y la base donde apoyarse para llegar a los nuevos compromisos que nos demandará la sociedad del futuro.

\section{REFERENCIAS BIBLIOGRÁFICAS}

Asheim, B. y Dunford, M. (1997): Regional Futures, Regional Studies, 31(5) 445-455.

Aydalot, P.(1986): Milieux innovateurs en Europe. París, Francia; GREMI.

Bagnasco, A.; Piselli, F.; Pizzorno, A.; Triglia, C. (2003). El capital social. Instrucciones de uso. Buenos Aires, Argentina; Fondo de Cultura Económica.

Becattini, G.(1979). Dal 'settore' industiale al 'distretto' industriale. Alcune considerazioni sull'unità d'indagine dell'economie industriale. Economia e Politica Industriale, 80, 25-48.

CAMAGNI,R (1989). Cambiamento tecnológico, milieu locale e reti di imprese: verso una teoria dinamica dello spazio economico. Economia e política industrial, 64, 209-236.

Caravaca, I. (1998). Los nuevos espacios ganadores y emergentes. EURE. Revista Latinoamericana de Estudios Urbano-Regionales, 24(73), 5-30.

Caravaca I., González, G. y Silva, R. (2003).Redes e innovación socio-institucional en sistemas productivos locales. Boletín de la Asociación de Geógrafos Españoles, 36, 103-115.

Consejo Social de la Universidad de Cádiz (2010). Una estructura consolidad de colaboración de universidad cd Cádiz. Recuperado de: http://www.consejosandalucia.org/premio/19-26.pdf 
F. Rufo/P. Ingelmo (7 de abril de 2013). Futuro y Decadencia. Diario de Cádiz. Recuperado de: http:// www.diariodecadiz.es/article/provincia/1496800/futuro/y/decadencia.html

Garofoli, G.(1992). Endogenous Development and Southern Europe. Aldershot, Hants., England; Avebury.

Instituto Fundación Hélice(2011). Informe del sector aeronáutico andaluz de 2011.Recuperado de: http://www.fundacionhelice.com/es/magazine/estudio-del-sector-2011.htm

Keeble, D. y Wilkinson, F. (1999). Collective learning and knowledge development in the evolution of regional clusters of high technology SMEs in Europe. Regional Studies, 33(4), 295-303.

Lawson, C.y Lorenz, E. (1999). Collective learning, tacit knowledge and regional innovative capacity. Regional Studies. 33(4), 305-317.

Maillat, D., Quevit, M. y Senn, L. (ed.). (1993). Réseaux d'innovation et milieux innovateurs: un pari pour le développement régional. Neuchâtel, Suiza. GREMI, EDES.

Maillat, D.(1995) Les millieux innovateurs, Sciences Humaines. 8, 41-42.

Maillat, D. y Kebir, L.,(1998). Learning region et systèmes territoriaux de production, Working Paper IRER, 9802a.

Méndez, R. y Caravaca. I. (1996): Organización industrial y territorio. Madrid, España; Síntesis.

Morgan, K. (1997). The Learning Region: Institutions, Innovation and Regional Renewal, Regional Studies, 31(5), 491-503.

Pecqueur, B. (1989). Le developpement local. Paris, Francia; Syros.

Powell, W. W.; Koput, K. W. y Smith-Doerr, L. (1996): Interorganizational collaboration and the locus of innovation: networks of learning in biotechnology. Administrative Science Quarterly, 41(1), 116-145.

Sforzi, F. (2002). Los distritos industriales ante el reto de la globalización. Geographicalia, 2002, 41, 5- 18.

Storper, M. (1993). Regional worlds of production: learning and innovation in the technology districts of France, Italy and the USA, Regional Studies, 27(5), 433-455

Redacción Asociación Pasión por volar. (15 de diciembre de 2010). Construcciones Aeronáuticas Sociedad Anónima-CASA. Recuperado de: http://www.pasionporvolar.com/construccionesaeronauticas-sociedad-anonima-casa/

Universidad de Cádiz (29 de septiembre de 2016). UCA, Junta, Airbus y Navantia firman el protocolo para el Centro de Innovación en Tecnologías de Fabricación Avanzada Recuperado de: http:// www.uca.es/es/cargarAplicacionNoticia.do?identificador=8723.

Universidad de Cádiz (8 de abril de 2017).UCA y EADS firman cinco convenios para impulsar la colaboración científico-tecnológica y la cooperación educativa. Recuperado de: http://www.uca. es/es/cargarAplicacionNoticia.do?identificador $=5798$

Universidad de Cádiz (21 de septiembre de 2015).. La Universidad de Cádiz genera más de 5.300 empleos y 414,2 M€ de impacto en su entorno. Recuperado de: http://www.uca.es/es/ cargarAplicacionNoticia.do?identificador=7909 
Universidad de Cádiz (5 de mayo de 2010). Dos empresas Campus de la UCA obtienen galardón en el II Premio Cádiz Innova. Recuperado de: http://www.uca.es/es/cargarAplicacionNoticia. do;jsessionid=85A30B0E1B1E033A E46F8676948E1EAB.wwwucaes 3?identificador=1050 )

Weber, Alfred (1929), Theory of Location of Industries, Chicago, EEUU; University of Chicago Press.

\section{Para citaciones:}

Reyes-Benítez, S. (2017). La Universidad como agente dinamizador del desarrollo territorial: el caso de la Universidad de Cádiz y el sector aeronáutico en España. Panorama Económico, 25, 3, pp. 399-422.

\section{AUTORA}

